\title{
Peritoneal Metastatic Cancer Stem Cells of Gastric Cancer with Partial Mesenchymal-Epithelial Transition and Enhanced Invasiveness in an Intraperitoneal Transplantation Model
}

\author{
Xiao-Hai Song $\mathbb{D},{ }^{1}$ Xin-Zu Chen $\mathbb{D}^{1},{ }^{1}$ Xiao-Long Chen $\mathbb{D}^{1},{ }^{1}$ Kai Liu, ${ }^{1}$ Wei-Han Zhang, \\ Xian-Ming Mo $\mathbb{D}^{2}$, and Jian-Kun $\mathrm{Hu} \mathbb{D}^{1}$ \\ ${ }^{1}$ Department of Gastrointestinal Surgery \& Laboratory of Gastric Cancer, West China Hospital, Sichuan University, Chengdu, China \\ ${ }^{2}$ Laboratory of Stem Cell Biology, West China Hospital, Sichuan University, Chengdu, China
}

Correspondence should be addressed to Xin-Zu Chen; chenxinzu@scu.edu.cn and Jian-Kun Hu; hujkwch@126.com

Received 17 December 2019; Revised 29 May 2020; Accepted 22 June 2020; Published 5 August 2020

Academic Editor: Tatsuya Toyokawa

Copyright ( $\odot 2020$ Xiao-Hai Song et al. This is an open access article distributed under the Creative Commons Attribution License, which permits unrestricted use, distribution, and reproduction in any medium, provided the original work is properly cited.

\begin{abstract}
Objectives. This preliminary study is aimed at enriching and isolating peritoneal metastatic cancer stem cells (pMCSCs) of gastric cancer and assessing their epithelial-mesenchymal transition (EMT) phenotype and invasiveness. Methods. Cancer stem cells of human gastric cancer (CSC-hGC) were previously isolated and transfected with green fluorescent protein and luciferase genes to validate the mouse model of peritoneal metastasis established via transplantation. The first and second generations ([G1] and [G2], respectively) of pMCSCs were isolated from intraperitoneally transplanted CSC-hGC (pMCSC-tGC) by spherical culture. CSC and EMT-related markers and regulators in the two generations of intraperitoneally transplanted tumors were examined by immunohistochemistry, immunofluorescence staining, and quantitative PCR. Cell mobility was examined by a transwell assay. Results. The nude mouse model of intraperitoneally transplanted CSC-hGC was successful in establishing sequential formation of peritoneal tumors and enrichment of pMCSCs. CD44 and CD54 were consistently expressed in the two generations of transplanted tumors. In vitro cell (migration) assays and immunocytofluorescence assays showed that in pMCSC$\mathrm{tGC}^{[\mathrm{G} 2]}$, E-cad, Survivin, and Vimentin expression was stable; $\alpha$-SMA expression was decreased; and OVOL2, GRHL2, and ZEB1 expression was increased. PCR analysis indicated that in pMCSC-tGC ${ }^{[\mathrm{G} 2]}$, the mRNA expression of E-cad, $\alpha$-SMA, MMP9, MMP2, and Vimentin was downregulated, while that of ZEB1, OVOL2, and GRHL2 was upregulated. In vivo tumor

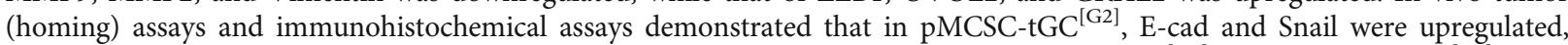

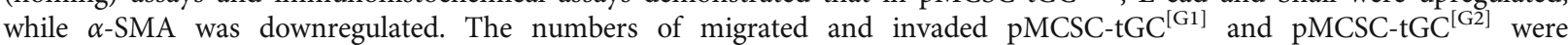
significantly higher than those of CSC-hGC in migration and invasion assays. Conclusions. pMCSCs might be a specific subpopulation that can be sequentially enriched by intraperitoneal transplantation. pMCSCs exhibited a tendency towards partial mesenchymal-epithelial transition, enhancing their invasiveness during homing and the formation of peritoneal tumors. However, these preliminary findings require validation in further experiments.
\end{abstract}

\section{Introduction}

Gastric cancer (GC) remains one of the most common malignancies, with high worldwide mortality [1]. In China, most GC patients are diagnosed with locally advanced or metastatic disease [2-4]. Peritoneal metastasis is found in up to $30 \%$ of GC patients at initial diagnosis and is the most common recurrence pattern after radical resection for locally advanced GC [5]. The median survival time of GC is only 3.1 months after diagnosis of peritoneal metastasis [6]. Cytoreductive surgery, systematic chemotherapy, and/or hyperthermic intraperitoneal chemotherapy are insufficient to improve the survival of these patients [7-9].

A minor subpopulation of tumor cells in a given tumor that have the potential for tumor initiation and tumor progression, called cancer stem cells (CSCs), has recently been identified $[10,11]$. CSCs are characterized by their abilities for self-renewal, tumorigenesis, and differentiation [12-14]. 
CSCs were identified first in hematologic malignancies [15] and then in various solid tumors $[11,16]$. Takaishi et al. isolated GC-initiating cells through identification of the cell surface marker CD44 [17]. We identified CSCs from the primary tumors and peripheral blood of GC patients through their expression of the surface markers CD44 and CD54 [18].

Epithelial cells exhibit clonal heterogeneity [19], which suggests the possibility of diverse clusters of initial CSCs. Therefore, it was hypothesized that a specific subpopulation, metastatic CSCs (MCSCs), might be responsible for metastasis [20]. The CSCs isolated from peripheral blood had stronger migratory and invasive abilities than those isolated from primary tumors [18]. A common feature of the tumor metastasis process is that specialized epithelial cells lose their adhesion and polarity, reorganize their cytoskeleton, and acquire a mesenchymal morphology and migration ability [21]. Moreover, compared with circulatory metastasis, peritoneal metastasis from GC might be a distinguishable pattern, especially regarding the homing of MCSCs. A cluster of peritoneal MCSCs (pMCSCs) may exist and function in GC peritoneal metastasis [22]. Therefore, we established a nude mouse model of peritoneal metastasis with CSCs of human GC (CSC-hGC) through intraperitoneal injection and aimed to enrich pMCSCs from intraperitoneally transplanted CSChGC (pMCSC-tGC) and characterize their stemness, tumorigenicity, and invasiveness.

\section{Materials and Methods}

2.1. CSC-hGC and Animals. CSC-hGC have previously been isolated from primary GC tissues, as reported elsewhere $[23,24]$. The specific markers of gastric CSCs are still controversial and under discussion. In our previous study, gastric CSCs were identified and isolated through their expression of the CSC markers CD44 and CD54. Our study indicated that the cells with a CD44(+)CD54(+) phenotype had characteristics of stem cells, as confirmed by serial transplantation in vivo and serial clone formation in vitro. Informed consent was obtained from patients who provided samples, and this study was approved by the Biomedical Ethics Committees of West China Hospital.

CSC-hGC expressed the surface markers CD44 and CD54 and formed xenografts with a morphology similar to that of the primary tumors. The CSC-hGC used in the present experiments were CSCs isolated from a poorly differentiated gastric adenocarcinoma (id: GCSC.112) (Figure 1(a)). Four-week-old female nude mice (Da Shuo Co., Ltd., Chengdu, China) were maintained under standard housing conditions at Sichuan University. The Institutional Biomedical Ethics Committee of West China Hospital approved the study protocol, and animal experiments were conducted according to the guidelines of the local Institutional Animal Care and Use Committee.

2.2. Lentiviral Transduction. CSC-hGC were detached with Accutase (Sigma, U.S.), seeded in a 96-well plate at a concentration of $4 \times 10^{4}$ cells $/ \mathrm{ml}$, and cultured to a confluence of between $50 \%$ and $70 \%$. Concentrated virus expressing green fluorescent protein and luciferase (GFP+LUC) (Hanbio
Biotechnology Co., Ltd., Shanghai, China) stored in a $80^{\circ} \mathrm{C}$ freezer was thawed on ice. Then, $100 \mu \mathrm{l}$ of serumfree medium and concentrated virus were added together to the 96-well plate. After infection for 72 hours, the fluorescence intensity of the CSC-hGC was observed under an inverted fluorescence microscope (Olympus, Japan). The CSC-hGC ${ }^{[\mathrm{GFP}+\mathrm{LUC}]}$ with the strongest fluorescence intensity and most robust growth were selected. Next, CSChGC ${ }^{[\mathrm{GFP}+\mathrm{LUC}]}$ with stable expression of GFP+LUC were obtained by puromycin selection.

2.3. Animal Model of Peritoneal Metastasis. Five mice were assigned to each group for separate experiments. Each mouse was injected with $1 \times 10^{3}, 1 \times 10^{4}, 1 \times 10^{5}$, or $1 \times$ $10^{6} \mathrm{CSC}-\mathrm{hGC} \mathrm{C}^{[\mathrm{GFP}+\mathrm{LUC}]}$ in $100 \mu \mathrm{l}$ of PBS at the lower midline into each side of the abdominal cavity. When $1 \times 10^{6}$ CSC-hGC ${ }^{[\mathrm{GFP}+\mathrm{LUC}]}$ were injected, tumors were formed from the intraperitoneally transplanted cells in the abdominal cavity of each mouse (Figure 1(d)). Thus, a dose of $1 \times 10^{6}$ CSC-hGC ${ }^{[\mathrm{GFP}+\mathrm{LUC}]}$ was adopted to establish the peritoneal metastasis model in our study. After validation of this mouse model two times via in vivo bioluminescence imaging, subsequent experiments based on the mouse model were performed according to the same procedure. Before the second intraperitoneal transplantation, the first-generation pMCSC-tGC (pMCSC-tGC ${ }^{[G 1]}$ ) were passaged for 3-4 generations.

2.4. In Vivo Bioluminescence Imaging. Mice were sedated with $4 \%$ chloral hydrate $(400 \mathrm{mg} / \mathrm{kg})$ and given a single intraperitoneal (i.p.) $150 \mathrm{mg} / \mathrm{kg}$ dose of D-luciferin (Nanjing Asian Chemical Co., Ltd., China) in PBS. Bioluminescence imaging with a CCD camera (Xenogen, U.S.) was initiated 10 minutes after injection of D-luciferin. The imaging time ranged from 1 to 60 seconds according to the amount of luciferase activity.

Bioluminescence was assessed in the region of interest (ROI), which was defined manually. All data are expressed in units of photon flux (photons $/ \mathrm{s} / \mathrm{cm}^{2} /$ steradian) and were collected and analyzed using the in vivo imaging system (Xenogen, U.S.).

2.5. Spherical Culture of $p M C S C$ - $t G C$. Four weeks later, all mice were sacrificed by $\mathrm{CO}_{2}$ inhalation. The transplanted GC tumors were harvested and washed 5 times with PBS containing $10 \%$ penicillin-streptomycin solution (HyClone, U.S.). Then, to obtain a single-cell suspension, the samples were cut into pieces and digested with collagenase IV in a $37^{\circ} \mathrm{C}$ incubator for $30-60$ minutes based on their size. Cell suspensions were collected after filtering through a $70 \mu \mathrm{m}$ filter. After centrifugation at $1000 \mathrm{r} / \mathrm{min}$ for 5 minutes, the cell pellet was collected and cultured in serum-free DME/F12 medium in a $\mathrm{CO}_{2}$ incubator at $37^{\circ} \mathrm{C}$. The cells isolated from the first generation of transplanted tumors derived from CSC-hGC in serum-free culture medium were defined as pMCSC-tGC ${ }^{[\mathrm{G} 1]}$. Similarly, pMCSC-tGC ${ }^{[\mathrm{G} 1]}$ were intraperitoneally injected to establish the second generation of transplanted tumors. Cell spheres isolated from the transplanted 


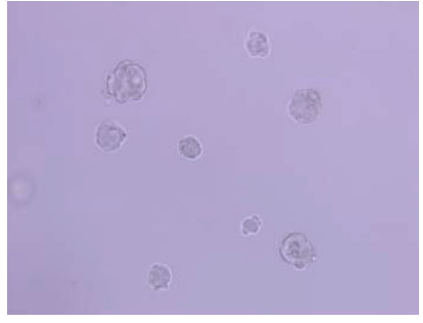

(a)

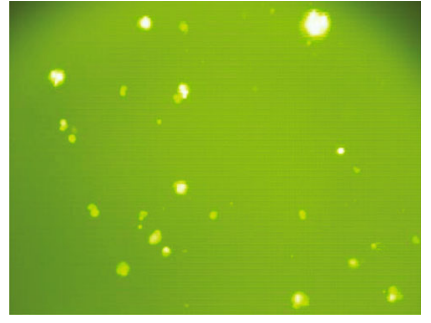

(b)

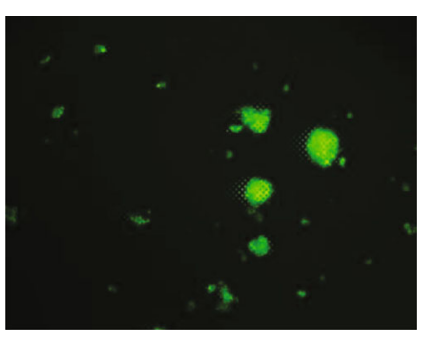

(c)

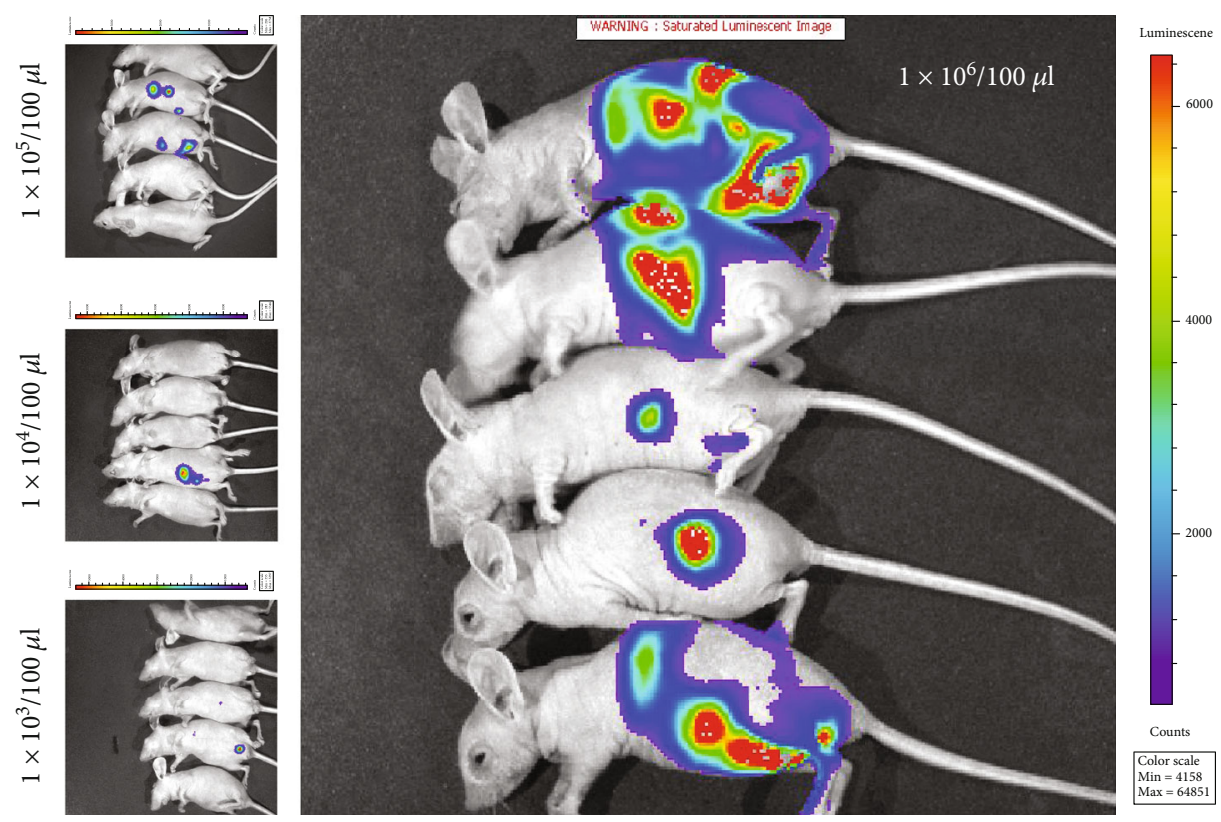

(d)

FIGURE 1: (a) The CSC-hGC sphere by subculture $(\times 200)$. (b, c) The successfully transfected CSC-hGC ${ }^{[G F P+L U C]}$ by immunocytofluorescence $(\times 200)$. (d) The bioluminescence imaging for nude mouse models injected intraperitoneally with $100 \mu l$ of $1 \times 10^{3}, 1 \times 10^{4}, 1 \times 10^{5}$, and $1 \times 10^{6}$ CSC-hGC ${ }^{[\mathrm{GFP}+\mathrm{LUC}]}$.

tumors derived from pMCSC-tGC ${ }^{[\mathrm{G} 1]}$ were defined as second-generation pMCSCs (pMCSC-tGC ${ }^{[\mathrm{G} 2]}$ ).

2.6. Morphological Assessment of Transplanted Tumors. The intraperitoneally transplanted tumors derived from CSChGC, pMCSC-tGC ${ }^{[\mathrm{G} 1]}$, and pMCSC-tGC ${ }^{[\mathrm{G} 2]}$ were excised by laparotomy, fixed with $10 \%$ buffered formalin, embedded in paraffin, sliced, and stained with hematoxylin-eosin (H\&E) (ZSGB-BIO, Beijing, China; Sigma, U.S.) before microscopic observation. Additionally, frozen sections were prepared from fresh tissues of transplanted tumors derived from CSC-hGC ${ }^{[G F P-L U C]}$, and the sections were then stained with DAPI (Sigma, U.S.). Fluorescence microscopy (Olympus, Japan) was used to visualize tumor cells.

2.7. Immunohistochemistry (IHC). The expression levels of CD44, CD54, E-cadherin (E-cad), $\alpha$ smooth muscle actin $(\alpha$-SMA), and Snail were examined in the two generations of transplanted tumors by IHC. The reagents used for immunohistochemical staining included a primary mouse antihuman E-cad monoclonal antibody (Abcam, Hong Kong, China), a primary rabbit anti-human $\alpha$-SMA monoclonal antibody (ProMab Biotechnologies, Inc., U.S.), a primary rabbit anti-human CD44 monoclonal antibody (eBioscience, U.S.), a primary rabbit anti-human Snail polyclonal antibody (Abcam, Hong Kong, China), a primary rabbit anti-human CD54 monoclonal antibody (Abcam, Hong Kong, China), and secondary goat anti-mouse and anti-rabbit antibodies (Life Technologies, U.S.). Two independent pathologists assessed the immunohistochemical staining, and multiple fields on each slide were selected for microscopic evaluation of the staining intensity and extent in each group. Conflicting assessments were resolved by discussion and agreement.

2.8. Immunocytofluorescence (ICF). Before ICF, cell spheres were digested into single cells with Accutase (Sigma, U.S.) at $37^{\circ} \mathrm{C}$ for 10 minutes and were then fixed with $4 \%$ paraformaldehyde, permeabilized with $0.5 \%$ Triton X-100, and stained with primary and secondary antibodies. First, cell slides were used for ICF, and frozen sections of cellular spheres were used in subsequent experiments, because the fluorescence of individual cells was too weak. For preparation of frozen sections, cell spheres were collected and washed with PBS three times. Then, the cell spheres were dehydrated with a $30 \%$ sucrose water solution for 18 hours. The sucrose water solution was discarded, and $200 \mathrm{ml}$ of Tissue OCT- 
Freeze Medium (Solarbio, U.S.) was mixed gently with the cell spheres at room temperature. The suspension was solidified in a $-20^{\circ} \mathrm{C}$ freezer and was then sliced into frozen sections. The frozen sections were stained with primary and secondary antibodies. Although different methods were used, the difference had a minimal effect on our measurements of relative protein expression levels. The expression of epithelial-mesenchymal transition- (EMT-) related markers and regulators, including E-cad, $\alpha$-SMA, matrix metalloproteinase 2 (MMP2), matrix metalloproteinase 9 (MMP9), Survivin, Vimentin, ZEB1, OVOL2, and GRHL2, in CSChGC, pMCSC-tGC ${ }^{[\mathrm{G} 1]}$, and pMCSC-tGC ${ }^{[\mathrm{G} 2]}$ was evaluated by ICF. The reagents used for ICF included primary mouse anti-human E-cad monoclonal antibodies (BD Biosciences, U.S.), primary rabbit anti-human MMP2 polyclonal antibodies (Abcam, Hong Kong, China), primary rabbit anti-human MMP9 monoclonal antibodies (Abcam, Hong Kong, China), a primary rabbit anti-human $\alpha$-SMA monoclonal antibody (Abcam, Hong Kong, China), a primary rabbit anti-human Survivin monoclonal antibody (Abcam, Hong Kong, China), a primary rabbit anti-human Vimentin monoclonal antibody (Cell Signaling Technology, U.S.), a primary mouse antihuman OVOL2 monoclonal antibody (Santa Cruz Biotechnology, U.S.), primary rabbit anti-human ZEB1 monoclonal antibodies (Abcam, Hong Kong, China), primary rabbit anti-human anti-GRHL2 polyclonal antibodies (SigmaAldrich, U.S.), and secondary goat anti-mouse and rabbit antibodies (Life Technologies, U.S.). Multiple fields were selected for microscopic evaluation of the staining intensity and extent in each group. The same methods were used for the 3 groups of samples.

2.9. Quantitative Real-Time PCR ( $q$-PCR). Total RNA was extracted with TRIzol (Life Technologies, U.S.) from CSChGC, pMCSC-tGC ${ }^{[\mathrm{G} 1]}$, and pMCSC-tGC ${ }^{[\mathrm{G} 2]}$. A TAKARA kit (TaKaRa Biotechnology Co., Ltd., Dalian, China) was used for quantitative real-time PCR. The $10 \mu \mathrm{l}$ reaction volume contained $5 \mu \mathrm{l}$ of SYBR premix $\mathrm{Ex} \mathrm{Taq}^{\mathrm{TM}}, 0.8 \mu \mathrm{l}$ of DNA template, $0.4 \mu \mathrm{l}$ of each primer, and $3.4 \mu \mathrm{l}$ of $\mathrm{dH}_{2} \mathrm{O}$. The thermal cycling conditions used for PCR were as follows: $95^{\circ} \mathrm{C}$ for 30 seconds and 40 cycles of 5 seconds at $95^{\circ} \mathrm{C}$ and 30 seconds at $60^{\circ} \mathrm{C}$. GAPDH was used as the internal control. All samples were measured in triplicate, and the PCR data were analyzed using the $2^{-\Delta \Delta \mathrm{Cq}}$ method. The same methods were used for the 3 groups of samples.

2.10. Transwell Assay. Before the transwell assay, cell spheres were digested into single cells with Accutase (Sigma, U.S.) at $37^{\circ} \mathrm{C}$ for 10 minutes, and the cells were then suspended in serum-free medium. A 24-well plate with transwell cell culture chambers (Corning, U.S.) was used. To acclimate the transwell cell culture chambers, $100 \mu \mathrm{l}$ of serum-free DME/F12 medium (HyClone, U.S.) and $600 \mu$ l of DMEM (HyClone, U.S.) supplemented with $10 \%$ fetal bovine serum (Gemini, U.S.) were added to the upper and lower chambers, respectively. Twelve hours later, the medium was removed from both chambers. Cells $\left(5 \times 10^{4}\right.$ cells/well $)$ were suspended in $100 \mu \mathrm{l}$ of serum-free medium and seeded in the upper chamber. In addition, $600 \mu \mathrm{l}$ of DMEM supplemented with $10 \%$ fetal bovine serum was added to the lower chamber. The cells remaining on the upper surface of the filter in the upper chamber after incubation for 18 hours at $37^{\circ} \mathrm{C}$ in a $\mathrm{CO}_{2}$ incubator were removed by wiping. The remaining cells were fixed and stained with Wright Giemsa Solution (Jian Cheng Technology Co., Ltd., Nanjing, China). Cell migration was quantified by counting the cells that migrated to the lower surface of the filter. Cells in nine fields per filter were counted under a light microscope at a magnification of $\times 400$. Most steps of the invasion experiment were similar to those described above for the migration experiment, except that Matrigel Matrix (BD Biosciences, U.S.) diluted with DME/F12 medium (1:5) was added to the upper chamber, and $1 \times$ $10^{5}$ cells/well were seeded. The same methods were used for the 3 groups of samples.

2.11. Statistical Analysis. Statistical analyses were performed using the SPSS software package (version 19.0; SPSS Inc., Chicago, IL, U.S.). Continuous variables are presented as the means \pm standard deviations (SDs), and independent $t$ -tests were used for comparisons. A probability $(p)$ value of less than 0.5 was considered to indicate a statistically significant difference. All $p$ values are two-sided.

\section{Results}

3.1. Establishment of the Mouse Model of Peritoneal Metastasis by CSC-hGC. CSC-hGC ${ }^{[\mathrm{GFP}+\mathrm{LUC}]}$ with stable expression of GFP+LUC were successfully generated (Figures 1(b)-1(d)). Animal experiments were completed with the designed sample size without adverse events other than tumorigenesis. Four weeks after intraperitoneal injection of CSC-hGC ${ }^{[\mathrm{GFP}+\mathrm{LUC}]}$, the mouse model of GC peritoneal metastasis was stably established with $1 \times 10^{6}$ injected cells (Figure 1(d)). CSC-hGC ${ }^{[\mathrm{GFP}+\mathrm{LUC}]}$ formed intraperitoneal tumors, as shown by H\&E staining (Figures 2(a)-2(e)). Additionally, fluorescence microscopy of frozen sections showed that tumor cells emitted green fluorescence (Figure 2(f)) and validated that the peritoneal tumors originated from CSC-hGC ${ }^{[\mathrm{GFP}+\mathrm{LUC}]}$. The subsequent experiments were performed effectively with that number of cells.

3.2. Stemness and Tumorigenicity of pMCSC- $t$ GC. pMCSC$\mathrm{tGC}^{[\mathrm{G} 1]}$ (id: pMCSC.112.p1) and pMCSC-tGC ${ }^{[\mathrm{G} 2]}$ (id: pMCSC.112.p2) were successfully isolated through spherical culture of the transplanted tumor cells (Figure 3). Both pMCSC-tGC ${ }^{[\mathrm{G} 1]}$ and pMCSC-tGC ${ }^{[\mathrm{G} 2]}$ formed dispersed peritoneal tumors after intraperitoneal injection. H\&E staining histologically confirmed the tumorigenicity of pMCSC$\mathrm{tGC}^{[\mathrm{G} 1]}$ and pMCSC-tGC ${ }^{[\mathrm{G} 2]}$ and demonstrated the similarly poor differentiation of transplanted tumors derived from CSC-hGC, pMCSC-tGC ${ }^{[\mathrm{G} 1]}$, and pMCSC-tGC ${ }^{[\mathrm{G} 2]}$ (Figure 3). In addition, the putative membrane markers of CSC-hGC, CD44 and CD54, were expressed in the first and second generations of transplanted tumors (Figure 3).

3.3. Phenotypes of Mesenchymal-Epithelial Transition (MET) of $p M C S C-t G C$. E-cad and Snail (evaluated in vivo to assess the homing status) were upregulated but $\alpha$-SMA was 


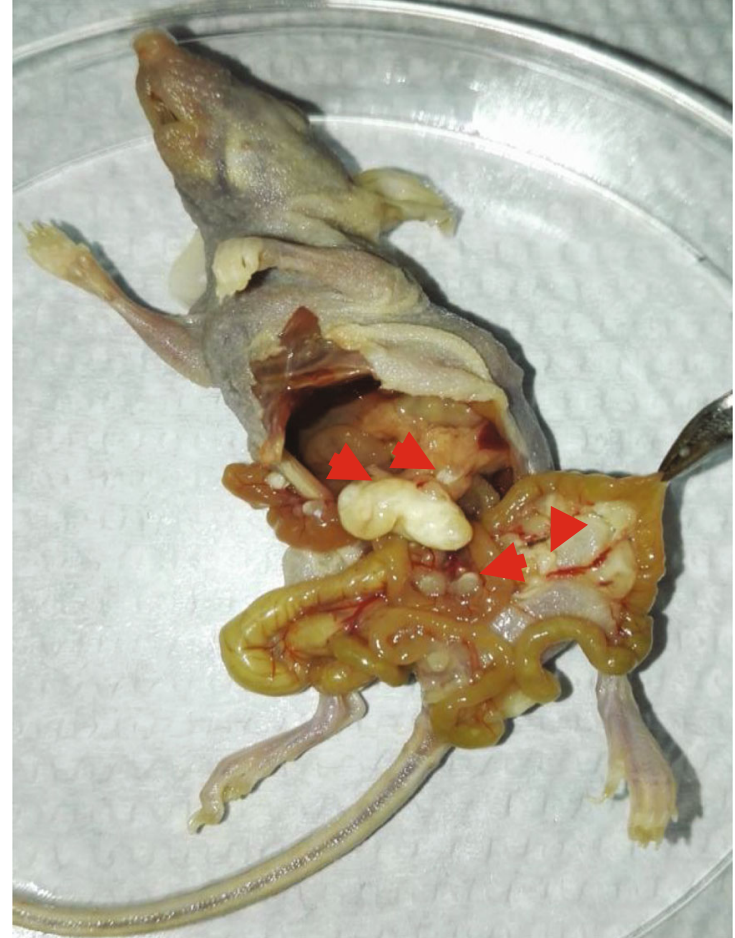

(a)

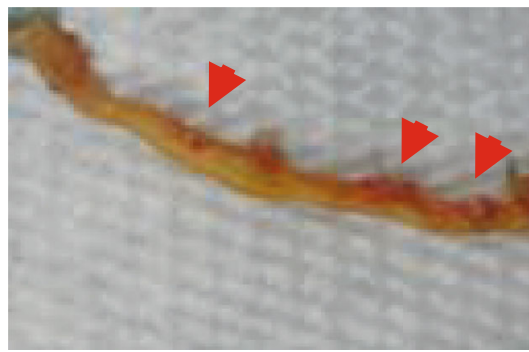

(c)

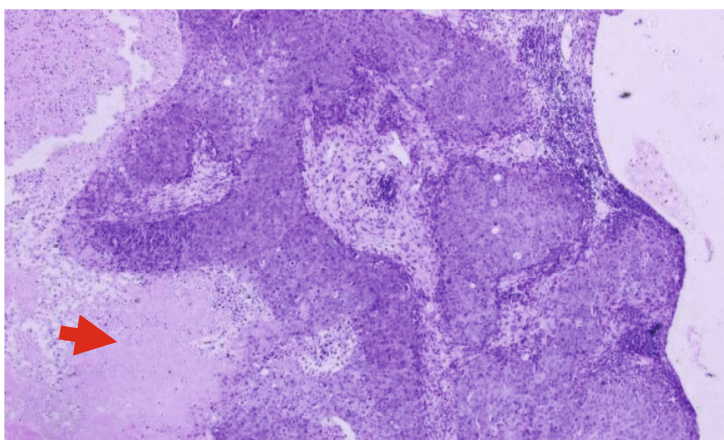

(e)

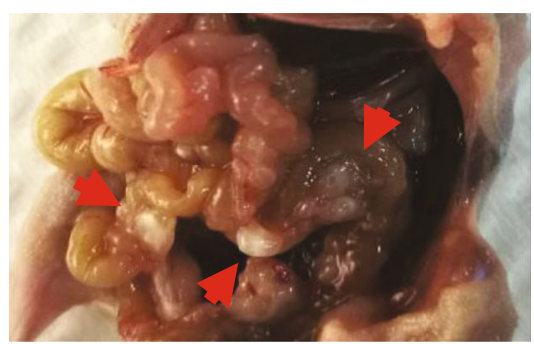

(b)

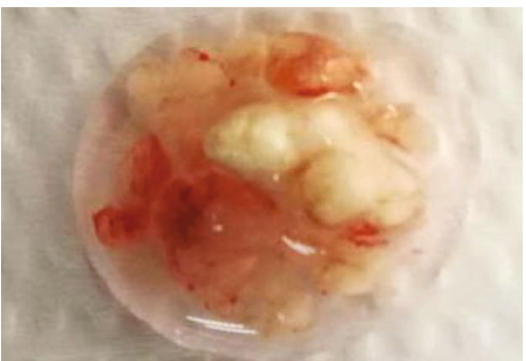

(d)

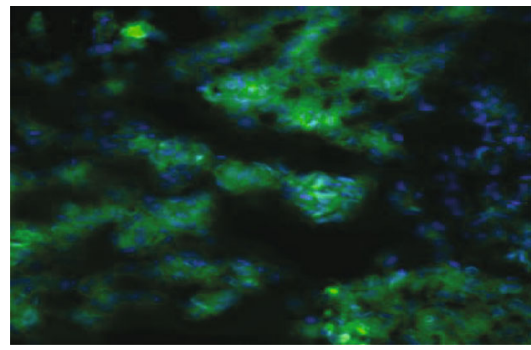

(f)

Figure 2: $(\mathrm{a}, \mathrm{b})$ In the sequential transplantation, gross anatomy generally showed that transplanted tumors, oval-shaped, with diverse size up to maximal $0.6 \mathrm{~cm}$, were located at the greater omentum and interintestinal space. (c) Those tumors distributed along mesenteric vessel with grain-like appearance. (d) When dissected, the tumor tissue presented creamy white color, irregular shape, and hard texture. (e) Histology by $\mathrm{H} \& \mathrm{E}$ staining $(\times 100)$ showed that tumor cells were clustered, and nuclei were large and with mitotic appearance. Necrotic regions were observed around tumor cell clusters, and no glandular structure was observed in tumor tissue with poor differentiation. (f) The frozen section under fluorescence microscope $(\times 200)$ showed the formation of intraperitoneal transplanted tumor from the CSC-hGC ${ }^{[\mathrm{GFP}+\mathrm{LUC}]}$ (green: tumor cells; blue: DAPI staining for nuclei). 

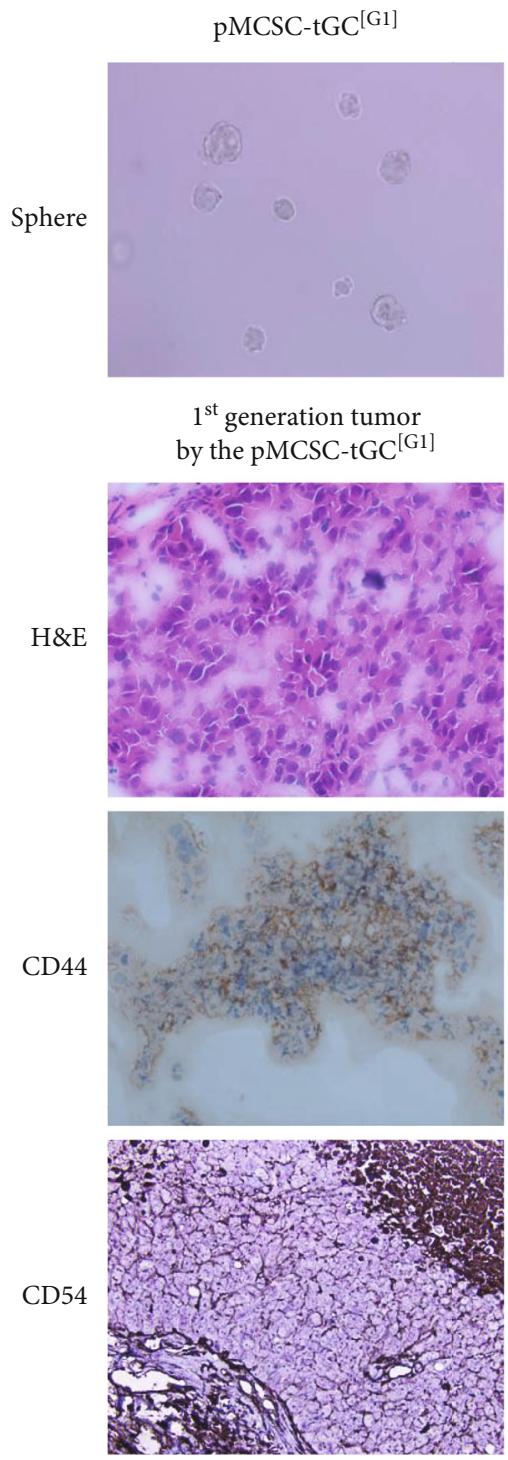

pMCSC-tGC ${ }^{[\mathrm{G} 2]}$

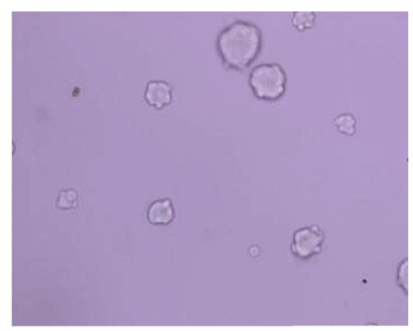

$2^{\text {nd }}$ generation tumor by the pMCSC-tGC ${ }^{[\mathrm{G} 2]}$
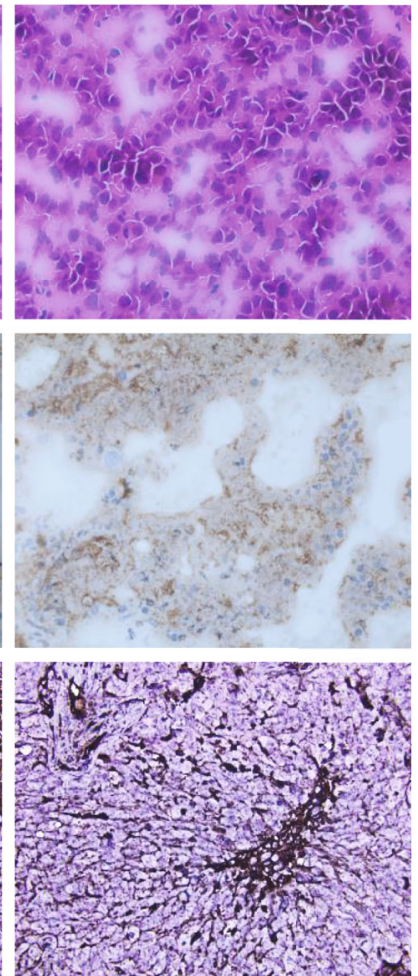

Figure 3: The tumor spheres $(\times 200)$ of the pMCSC-tGC ${ }^{[\mathrm{G} 1]}$ and $\mathrm{pMCSC}-\mathrm{tGC}^{[\mathrm{G} 2]}$ and correspondingly the H\&E staining $(\times 200)$ and immunohistochemistry of CD44 and CD54 $(\times 200)$ for their intraperitoneally transplanted tumors.

downregulated in the transplanted tumors derived from pMCSC-tGC ${ }^{[\mathrm{G} 2]}$ compared with the transplanted tumors derived from pMCSC-tGC ${ }^{[\mathrm{G} 1]}$ (Figure 4). The ICF results showed that after sequential formation of intraperitoneal tumors, E-cad, Survivin, Vimentin, MMP2, and MMP9 (evaluated in vitro to assess the cell status) exhibited stable expression in pMCSC-tGC cells, $\alpha$-SMA exhibited decreased expression. However, OVOL2, GRHL2, and ZEB1 exhibited increased expression in pMCSC-tGC ${ }^{[\mathrm{G} 2]}$ (Figure 5). RTPCR of pMCSC-tGC cells (in vitro) revealed that compared with the corresponding levels in CSC-hGC, the expression levels of mRNAs encoding E-cad $(p<0.0001)$, MMP9 $(p=0.0006), \alpha$-SMA $(p=0.0127)$, Vimentin $(p=0.0413)$, and MMP2 $(p=0.0004)$ were decreased in pMCSC-tGC ${ }^{[\mathrm{G} 2]}$, while the expression levels of mRNAs encoding ZEB1 $(p=0.0039)$, OVOL2 $(p=0.0025)$, and RGHL2 $(p=0.0252)$ were increased in pMCSC-tGC ${ }^{[\mathrm{G} 2]}$ (Figure 6).
3.4. Enhanced Migration and Invasion of pMCSC- $t G C$. The transwell assay results demonstrated that the numbers of migrated $(92.3 \pm 2.5$ vs. $62.0 \pm 2.0, p<0.001)$ and invaded $(54.7 \pm 1.2$ vs. $28.7 \pm 1.2, p<0.001)$ pMCSC-tGC ${ }^{[G 1]}$ were higher than those of CSC-hGC. Similarly, the numbers of migrated $(91.0 \pm 2.6$ vs. $62.0 \pm 2.0, p<0.001)$ and invaded $(52.7 \pm 2.1$ vs. $28.7 \pm 1.2, p<0.001)$ pMCSC-tGC ${ }^{[\mathrm{G} 2]}$ were higher than those of CSC-hGC (Figure 7).

\section{Discussion}

In this preliminary study, we proposed a novel hypothesis regarding the mechanism underlying peritoneal metastasis of GC, postulating that it is derived from a potential cluster of pMCSCs. To our knowledge, this study is the first to investigate the effect of pMCSCs on peritoneal metastasis. We also successfully established a nude mouse model of 


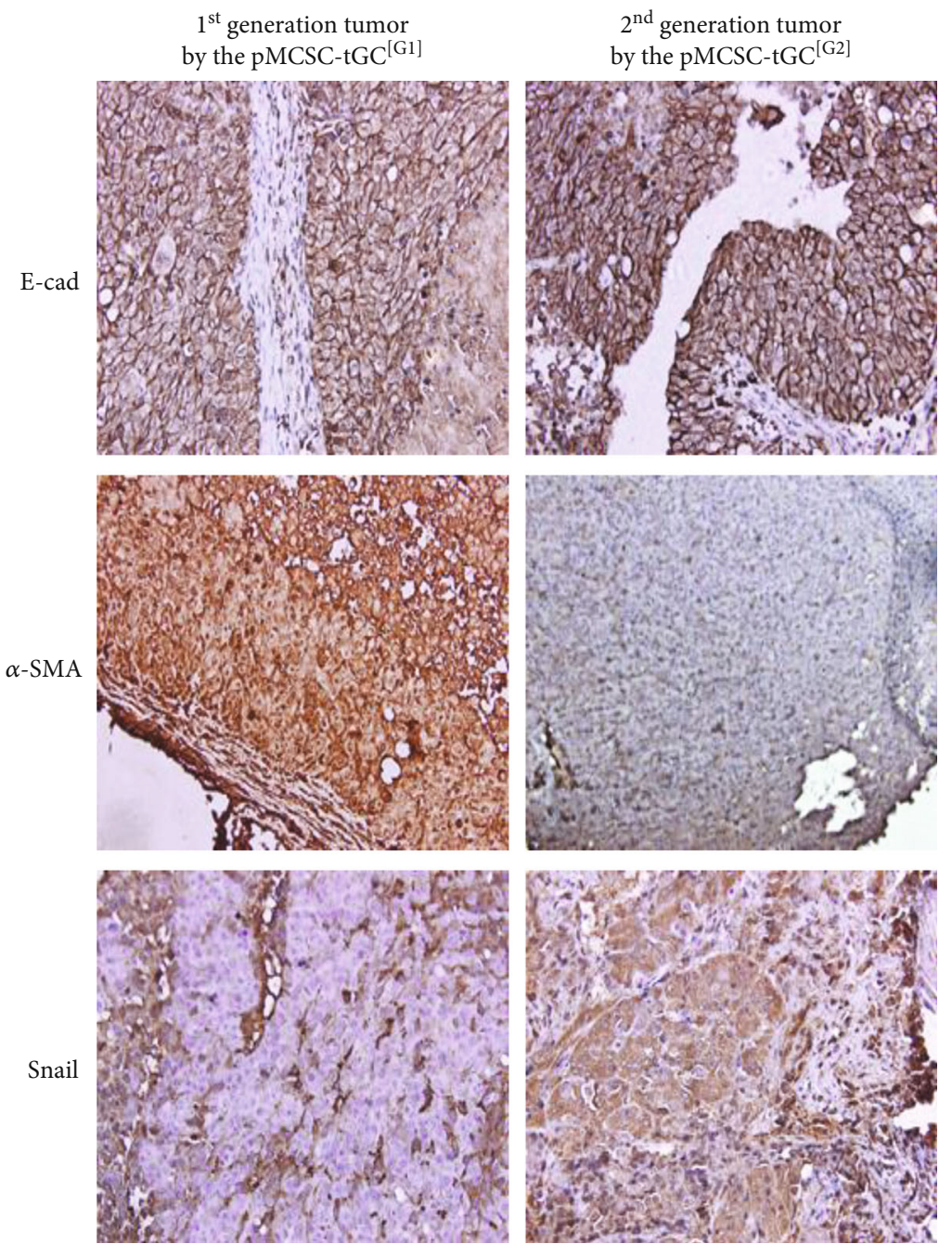

FIGURE 4: Immunohistochemistry of E-cad, $\alpha$-SMA, and Snail $(\times 200)$ for the two generations of intraperitoneally transplanted tumors from the pMCSC-tGC ${ }^{[\mathrm{G} 1]}$ and pMCSC-tGC ${ }^{[\mathrm{G} 2]}$.

peritoneal metastasis through intraperitoneal injection of CSC-hGC. In this animal model, we performed sequential intraperitoneal transplantation and isolated the first- and second-generation pMCSC-tGC. The stemness and tumorigenicity of pMCSC-tGC ${ }^{[\mathrm{G} 1]}$ and pMCSC-tGC ${ }^{[\mathrm{G} 2]}$ well succeeded to the CSC-hGC. Sequential intraperitoneal transplantation possibly enhanced the partial epithelial phenotypes of pMCSC-tGC and strengthened their migratory and invasive capabilities.

Researchers have isolated gastric CSCs from various GC cell lines and from tumor tissues and peripheral blood of GC patients. Different CSC markers, such as Lgr5, CD44, CD133, CD54, DLL4, and CD24, have been reported [2326]. However, the specific CSC markers are still controversial and under discussion. In our previous study, gastric CSCs were identified and isolated from tumor tissues and peripheral blood of gastric adenocarcinoma patients through the expression of the CSC markers CD44 and CD54 [18]. Our study indicated that the cells with a CD44(+)CD54(+) phenotype had characteristics of stem cells. First, those cells had the capabilities for sphere formation and self-renewal in serum-free medium, while in medium containing serum, the number of cells with the CD44(+)CD54(+) phenotype was decreased. Second, the results of animal experiments indicated that these cells had strong tumorigenic properties and could form tumors continuously. In addition, subcutaneous xenografts derived from those cells in nude mice exhibited histologic features similar to those of the primary GC tissue. Compared with GC cell lines, those cells had stronger invasion, metastasis, self-renewal, and proliferation abilities. Thus, we believed that the clusters of CD44(+)CD45(+) cells isolated from tumor tissues and peripheral blood of gastric adenocarcinoma patients were gastric CSCs.

Previous studies have investigated the mechanism underlying peritoneal metastasis of GC in vitro [27, 28]. Several models of peritoneal metastasis have been established by orthotopic implantation techniques and intraperitoneal injection of human GC cell lines [29-33]. We established a nude mouse model via intraperitoneal injection of CSChGC. Through in vivo bioluminescence or fluorescence imaging, gene and cell marker expression can be observed 


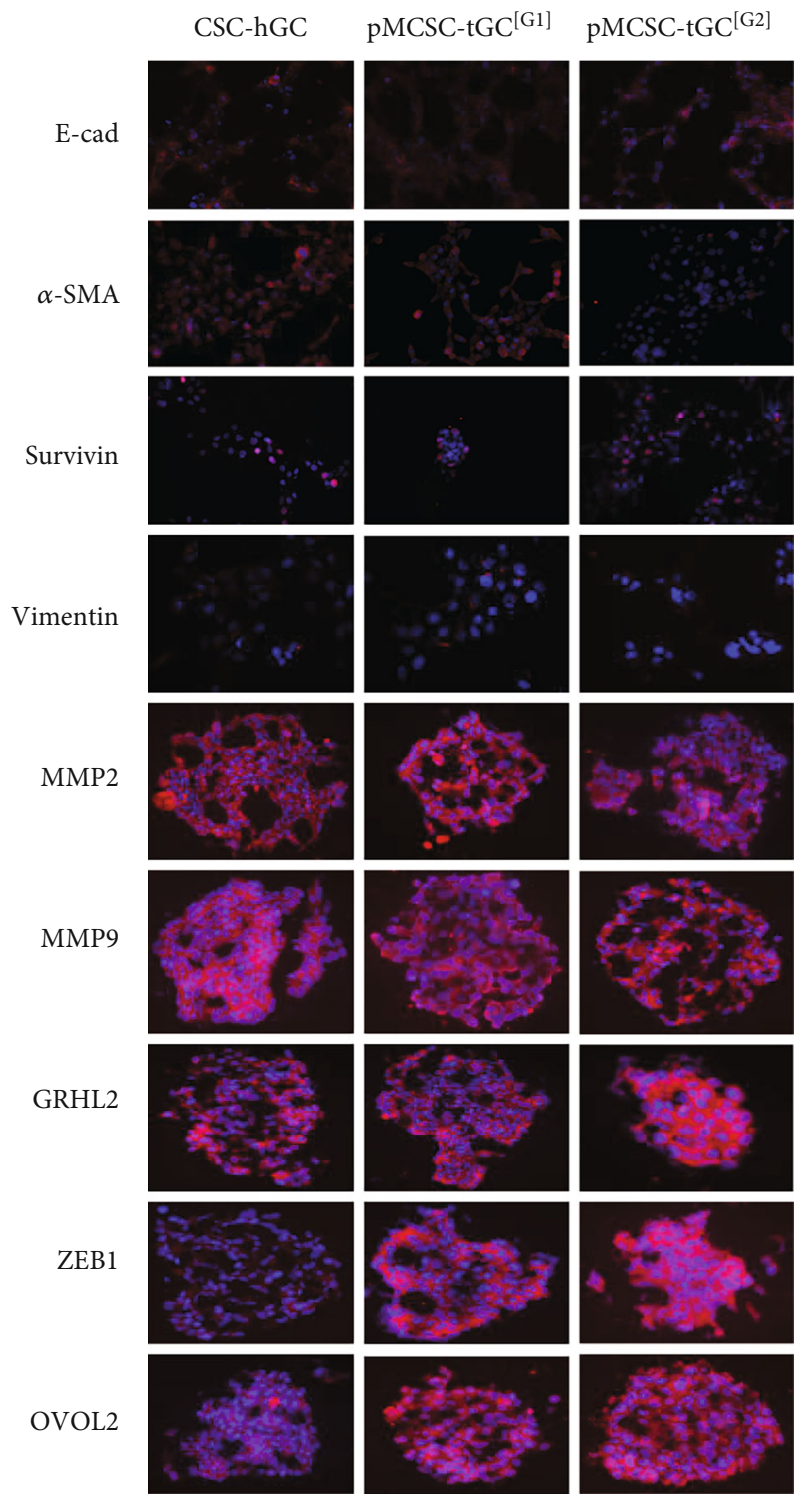

FIGURE 5: Immunocytofluorescence of E-cad, $\alpha$-SMA, Survivin, Vimentin, MMP2, MMP9, GRHL2, ZEB1, and OVOL2 $(\times 400)$ for the CSC-hGC, pMCSC-tGC ${ }^{[\mathrm{G} 1]}$, and pMCSC-tGC ${ }^{[\mathrm{G} 2]}$ cells.

in vivo in living animals $[34,35]$. GFP and LUC have already been widely used in this system for evaluating single cells in vitro and for functional testing in vivo [36-39]. In this study, we monitored and validated the tumorigenicity of CSCs and pMCSCs via in vivo bioluminescence imaging.

CSCs have been implicated not only in initiating and sustaining primary tumor growth but also in driving the seeding and formation of metastases at distal sites [40-44]. The existence of the MCSC population has been demonstrated in various human cancers. For example, both CD133(+)CXCR4(+) and CD133(+)CXCR4(-) pancreatic CSCs can form tumors, but only the CD133(+)CXCR4(+) subset has migration capability [45]. In colorectal cancer, the metastatic phenotype is determined by a distinct subpopulation of CD26(+) CSCs [46]. Associations between MCSCs and metastasis have also been found in several types of carcinomas [45-47]. In this study, interestingly, the identified pMCSC-tGC initially exhibited or acquired enhanced migratory and invasive capabilities after intraperitoneal transplantation. The Ras-MAPK signaling pathway is involved in tumor cell migration and invasion by regulating ECM degradation and mediating cell adhesion and movement [48, 49]. However, whether the Ras-MARK signaling pathway was activated under the conditions established in this study is unclear, and this possibility requires further study and discussion. pMCSCs can be enriched from the CSC-hGC population through this animal model, implying that peritoneal metastasis of GC may be associated with clusters of pMCSCs.

EMT is often activated during cancer invasion and metastasis and is characterized by loss of epithelial differentiation and a shift towards a mesenchymal phenotype [50, 51]. EMT is potentially associated with a set of markers, such as members of the cadherin family and $\alpha$-SMA. E-cad, an epithelial marker, is the most commonly investigated marker and has been found to be strongly associated with the EMT process by maintaining the stability of intercellular junctions. Expression of E-cad negatively correlates with EMT and cell mobility. Another member of the cadherin family, P-cad, acts as a double-edged sword, as either a tumor suppressor or oncogenic protein [52]. Moreover, P-cad overexpression is associated with cancer cell invasion and metastatic dissemination [52]. However, the functional role of $\mathrm{P}$-cad was proven to be dependent on the cellular context of E-cad in a breast cancer model [53]. Therefore, in the present study, only E-cad and not P-cad was evaluated to assess the tendency towards EMT.

The results of IHC (evaluated in in vivo tumors to assess the homing status) indicated that the expression of E-cad in pMCSC-tGC ${ }^{[\mathrm{G} 2]}$ was upregulated, but the ICF results (evaluated in vitro in free cells to assess the migration status) indicated that E-cad expression in these cells was stable, suggesting an epithelial phenotype indicating a tendency towards MET. However, the consistent phenotype of $\alpha$ SMA downregulation in pMCSC-tGC ${ }^{[\mathrm{G} 2]}$ observed in the immunohistochemical, ICF, and RT-PCR assays indicated the tendency towards MET, as did the downregulation of Vimentin. The transition from an epithelial to a mesenchymal phenotype involves downregulation of epithelial markers such as E-cad and upregulation of mesenchymal markers such as Vimentin and $\alpha$-SMA [54-56]. Therefore, our major findings suggested that pMCSC-tGC ${ }^{[\mathrm{G} 2]}$ potentially exhibited an enhanced epithelial phenotype, namely, a tendency towards a transition from a mesenchymal to an epithelial phenotype.

The key molecular markers of pMCSC-tGC, E-cad and $\alpha$ SMA, were evaluated under different growth conditions. We hypothesized that in vitro free cells (migration-mesenchymal phenotype) and in vivo tumors (homing-epithelial phenotype) might exhibit differential expression of EMT markers. Our results generally supported this hypothesis. Two generations of cells, pMCSC-tGC ${ }^{[\mathrm{G} 1]}$ and pMCSC-tGC ${ }^{[\mathrm{G} 2]}$, exhibited stable or downregulated expression of E-cad as in vitro free cells (mesenchymal status) but upregulated expression of E-cad as in vivo tumor cells (epithelial status). Similarly, 


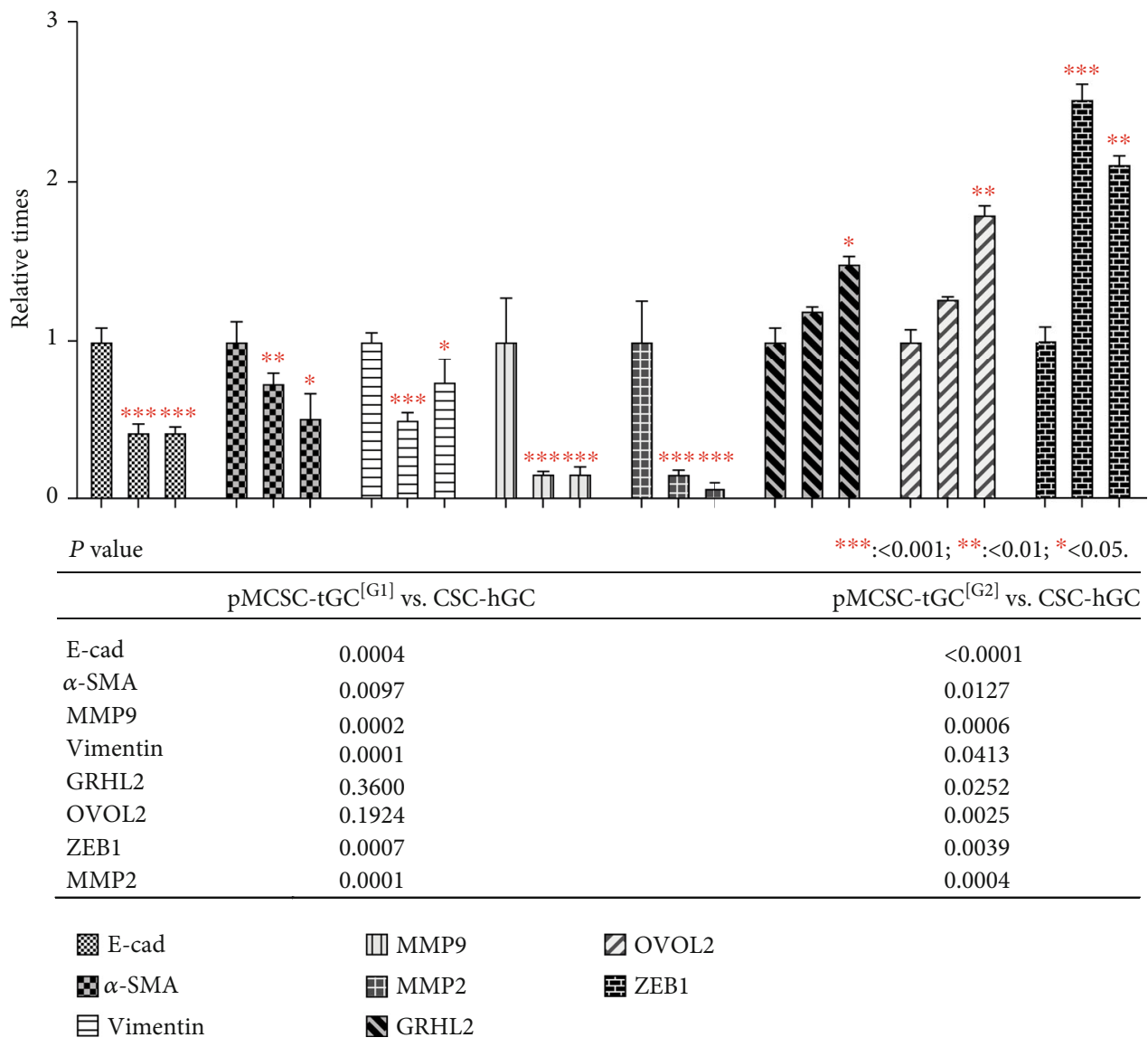

FIGURE 6: Quantitative real-time PCR of E-cad, $\alpha$-SMA, MMP9, Vimentin, GRHL2, OVOL2, ZEB1, and MMP2 for the CSC-hGC, pMCSC$\mathrm{tGC}^{[\mathrm{G} 1]}$, and pMCSC-tGC ${ }^{[\mathrm{G} 2]}$ cells.
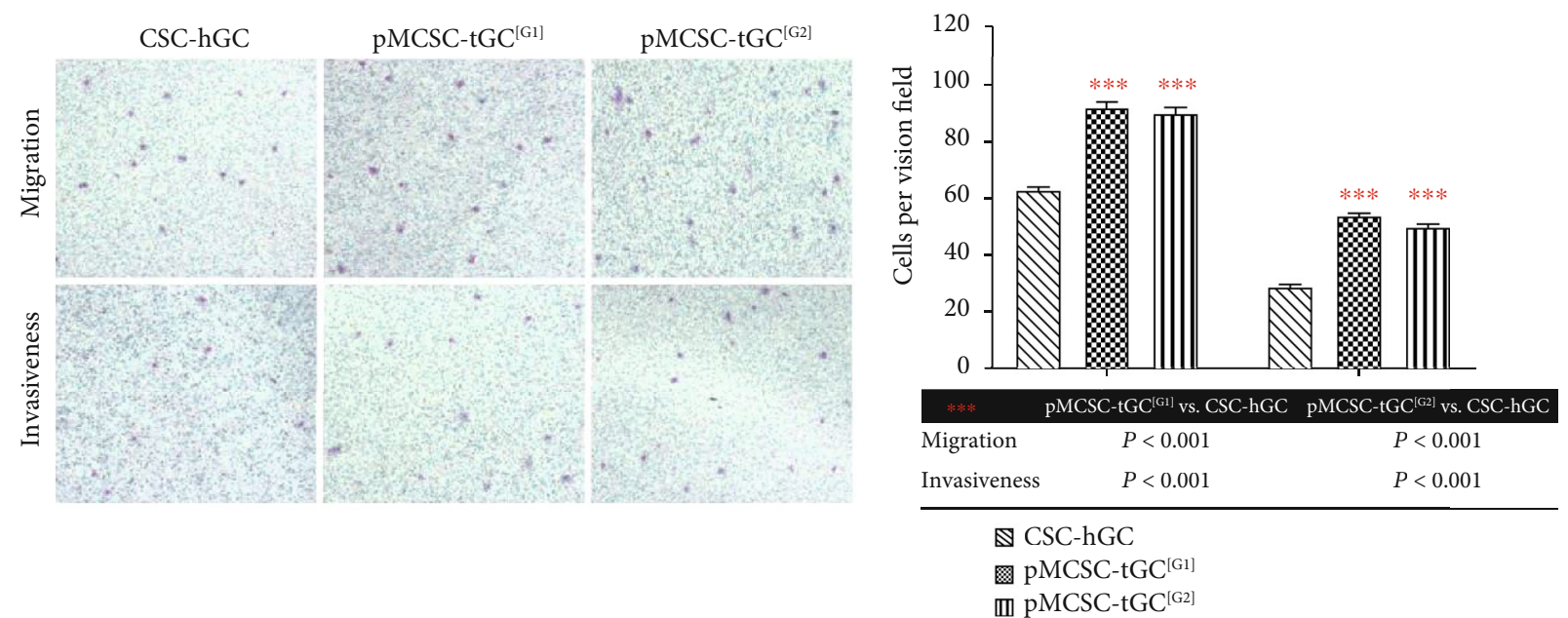

Figure 7: Transwell assays $(\times 400)$ for the cell mobility of the CSC-hGC, pMCSC-tGC ${ }^{[\mathrm{G} 1]}$, and pMCSC-tGC ${ }^{[\mathrm{G} 2]}$.

$\alpha$-SMA, a mesenchymal marker, was downregulated in in vivo tumor cells (epithelial status), consistent with the upregulation of E-cad.

Additionally, members of the MMP family can induce EMT and promote tumor cell mobilization by degrading the basement membrane and destroying intercellular junc- tions [54, 57]. In our study, the expression of MMP2 and MMP9 was downregulated, as determined by PCR. These findings also indicated that MET might occur during homing. Finally, not only is Snail a key inducer of EMT, but it also has EMT-independent functions in cell survival and stem cell biology [58]. The upregulation of Snail observed here may be 
inferred to be a function of stemness maintenance rather than EMT.

Recently, MET has been observed in metastases of most human cancers [59-61]. The progression of solid tumors is accompanied by EMT, through which tumor cells acquire enhanced invasive and metastatic capabilities. In the appropriate microenvironments of target organs, disseminated tumor cells must undergo the inverse transition, i.e., MET, for successful metastasis. Initiation of tumor growth at the secondary site has been proposed to be a dynamic process, and both EMT and MET have been indicated to be the driving forces of metastasis in different stages [51, 61, 62]. Chaffer et al. demonstrated the importance of the epithelial phenotype in secondary tumor formation by a cell line from human transitional carcinoma of the bladder [63]. In addition, previous studies showed that MET is necessary for the colonization and metastasis of differentiated cancers and that cancer cell growth is arrested if EMT is not reversed to MET [64, 65].

EMT/MET has long been viewed primarily as a binary process [66-68]. However, recent studies have shown that during the transition between the epithelial (E) and mesenchymal (M) phenotypes, cells can acquire a stable hybrid epithelial/mesenchymal (E/M) phenotype or a partial EMT phenotype. These hybrid E/M cells that coexpress epithelial and mesenchymal markers have mixed epithelial and mesenchymal properties $[69,70]$. The hybrid E/M phenotype is related to the stemness of cancer cells, and CSCs in several cancers (such as breast, prostate, lung, and colorectal cancers) have been found to coexpress epithelial markers and mesenchymal markers, suggesting a hybrid E/M phenotype [71-76]. In the present study, we found that epithelial markers and mesenchymal markers were expressed simultaneously in CSC-hGC, as well as in pMCSC-tGC ${ }^{[\mathrm{G} 1]}$ and pMCSC-tGC ${ }^{[\mathrm{G} 2]}$, suggesting that all of these cells had a hybrid E/M phenotype. In addition, compared to CSCs, both pMCSC-tGC ${ }^{[\mathrm{G} 1]}$ and pMCSC-tGC ${ }^{[\mathrm{G} 2]}$ showed a decreasing trend in the levels of epithelial and mesenchymal markers as measured by quantitative real-time PCR, indicating that pMCSC-tGC were more likely to be hybrid E/M ones. Previous studies reported that compared to the pure $\mathrm{M}$ or $\mathrm{E}$ phenotype, the hybrid E/M phenotype and the partial E/M phenotype are correlated with increased invasive and metastatic potential [69-72]. Our results showed that pMCSC$\mathrm{tGC}^{[\mathrm{G} 1]}$ and pMCSC-tGC ${ }^{[\mathrm{G} 2]}$ acquired enhanced migratory and invasive capabilities, further showing that pMCSC-tGC might acquire a hybrid E/M phenotype. EMT can be induced by overexpression of EMT-related transcription factors (termed EMT-TFs), such as Snail and ZEB1/2 [77-79], while MET can also be induced by a set of MET-TFs such as OVOL1/2 and RGHL2 [78-80]. Recent studies demonstrated that ZEB1, GRHL2, and OVOL2 are components of the core EMT regulatory circuit and play important roles in regulating EMT dynamics and stabilizing the $\mathrm{E}, \mathrm{M}$, and $\mathrm{E} / \mathrm{M}$ phenotypes [70, 76-80]. Complete MET has been reported to require overexpression of MET-TFs such as GRHL2 and/or OVOL2 and knockdown of the EMT-TFs such as ZEB1 $[70,78]$. To further investigate the complete or partial MET of pMCSC-tGC, we measured the changes in the expression of ZEB1, OVOL2, and GRHL2 in CSC-hGC, pMCSC-
$\mathrm{tGC}^{[\mathrm{G} 1]}$, and pMCSC-tGC ${ }^{[\mathrm{G} 2]}$. ZEB1, GRHL2, and OVOL2 were upregulated in pMCSC-tGC, and we considered that GRHL2 and OVOL2 probably promote MET, whereas ZEB1 might inhibit MET during peritoneal metastasis of CSC-hGC, which also suggested that pMCSC-tGC exhibit partial MET [70, 78]. EMT and MET can be regulated by numerous other mechanisms in addition to transcriptional control mechanisms, such as long noncoding RNAs, microRNAs, epigenetic modifications, alternative splicing, and posttranslational alterations in protein stability [81-87]. These mechanisms can differ among cell types or tissues of origin [76]. Our preliminary study showed that homing to the peritoneum through partial MET was necessary for pMCSC-tGC, and this finding requires further investigation.

In conclusion, a mouse model of GC peritoneal metastasis based on CSC-hGC transplantation was established, and pMCSC-tGC were isolated by sequential intraperitoneal transplantation. This strategy is a novel approach for investigating and understanding the potential mechanism of $\mathrm{GC}$ peritoneal metastasis. Compared to CSC-hGC, pMCSCtGC had an enhanced invasive capability, possibly due to partial MET during homing and the formation of peritoneal colonies. pMCSCs may be the driving force underlying peritoneal metastasis of GC. Further investigations are needed to gain a clearer understanding of the complex process of the EMT-MET shift during peritoneal metastasis, and these preliminary findings require validation in further experiments.

\section{Data Availability}

The data can be available by the email chenxinzu@scu.edu.cn.

\section{Additional Points}

Core Tip. Cancer stem cells (CSCs) of human gastric cancer (GC) (termed CSC-hGC) were previously isolated. The nude mouse model of intraperitoneally transplanted CSC-hGC was successful in establishing sequential formation of peritoneal tumors and enrichment of pMCSCs. pMCSCs might be a specific subpopulation that can be sequentially enriched in an intraperitoneal transplantation model. pMCSCs exhibited a tendency towards partial mesenchymal-epithelial transition, enhancing their invasiveness during homing and the formation of peritoneal tumors. However, these preliminary findings require validation in further experiments.

\section{Conflicts of Interest}

The authors declare that they have no conflicts of interest.

\section{Authors' Contributions}

Song $\mathrm{XH}$ and Chen $\mathrm{XZ}$ contributed equally as co-first authors in this work.

\section{Acknowledgments}

Support was given by the National Natural Science Foundation of China (NSFC, No. 81301866) for Chen XZ; the Outstanding Young Scientific Scholarship Foundation of 
Sichuan University, from the Fundamental Research Funds for the Central Universities of China (No. 2015SCU04A43) for Chen XZ; and the $1 \bullet 3 \bullet 5$ Project for Disciplines of Excellence, West China Hospital, Sichuan University (No. ZY2017304) for $\mathrm{Hu} \mathrm{JK}$.

\section{References}

[1] J. Ferlay, I. Soerjomataram, R. Dikshit et al., "Cancer incidence and mortality worldwide: sources, methods and major patterns in GLOBOCAN 2012," International Journal of Cancer, vol. 136, no. 5, pp. E359-E386, 2015.

[2] X. Z. Chen, Y. Liu, R. Wang, W. H. Zhang, and J. K. Hu, "Improvement of cancer control in mainland China: epidemiological profiles during the 2004-10 National Cancer Prevention and Control Program," Lancet, vol. 388, p. S40, 2016.

[3] R. Wang and X.-Z. Chen, "High mortality from hepatic, gastric and esophageal cancers in mainland China: 40 years of experience and development," Clinics and Research in Hepatology and Gastroenterology, vol. 38, no. 6, pp. 751-756, 2014.

[4] X. Z. Chen, W. H. Zhang, and J. K. Hu, "A difficulty in improving population survival outcome of gastric cancer in mainland China: low proportion of early diseases," Medical Oncology, vol. 31, no. 12, 2014.

[5] Y. Yonemura, Y. Endou, M. Shinbo et al., "Safety and efficacy of bidirectional chemotherapy for treatment of patients with peritoneal dissemination from gastric cancer: selection for cytoreductive surgery," Journal of Surgical Oncology, vol. 100, no. 4, pp. 311-316, 2009.

[6] G. Glockzin, H. J. Schlitt, and P. Piso, "Peritoneal carcinomatosis: patients selection, perioperative complications and quality of life related to cytoreductive surgery and hyperthermic intraperitoneal chemotherapy," World Journal of Surgical Oncology, vol. 7, no. 1, p. 5, 2009.

[7] T. Yoshikawa, M. Kanari, A. Tsuburaya et al., "Should gastric cancer with peritoneal metastasis be treated surgically?" Hepato-Gastroenterology, vol. 50, no. 53, pp. 1712-1715, 2003.

[8] C. Li, M. Yan, J. Chen et al., "Surgical resection with hyperthermic intraperitoneal chemotherapy for gastric cancer patients with peritoneal dissemination," Journal of Surgical Oncology, vol. 102, no. 5, pp. 361-365, 2010.

[9] Y. Fujiwara, S. Takiguchi, K. Nakajima et al., "Neoadjuvant intraperitoneal and systemic chemotherapy for gastric cancer patients with peritoneal dissemination," Annals of Surgical Oncology, vol. 18, no. 13, pp. 3726-3731, 2011.

[10] E. Charafe-Jauffret, C. Ginestier, F. Iovino et al., "Breast cancer cell lines contain functional cancer stem cells with metastatic capacity and a distinct molecular signature," Cancer Research, vol. 69, no. 4, pp. 1302-1313, 2009.

[11] C. T. Jordan, M. L. Guzman, and M. Noble, "Cancer stem cells," The New England Journal of Medicine, vol. 355, no. 12, pp. 1253-1261, 2006.

[12] S. P. McDermott and M. S. Wicha, "Targeting breast cancer stem cells," Molecular Oncology, vol. 4, no. 5, pp. 404-419, 2010.

[13] T. Lapidot, C. Sirard, J. Vormoor et al., "A cell initiating human acute myeloid leukaemia after transplantation into SCID mice," Nature, vol. 367, no. 6464, pp. 645-648, 1994.

[14] T. Reya, S. J. Morrison, M. F. Clarke, and I. L. Weissman, "Stem cells, cancer, and cancer stem cells," Nature, vol. 414, no. 6859, pp. 105-111, 2001.
[15] D. Bonnet and J. E. Dick, "Human acute myeloid leukemia is organized as a hierarchy that originates from a primitive hematopoietic cell," Nature Medicine, vol. 3, no. 7, pp. 730737, 1997.

[16] L. Ricci-Vitiani, D. G. Lombardi, E. Pilozzi et al., "Identification and expansion of human colon-cancer-initiating cells," Nature, vol. 445, no. 7123, pp. 111-115, 2007.

[17] S. Takaishi, T. Okumura, S. Tu et al., "Identification of gastric cancer stem cells using the cell surface marker CD44," Stem Cells, vol. 27, no. 5, pp. 1006-1020, 2009.

[18] T. Chen, K. Yang, J. Yu et al., "Identification and expansion of cancer stem cells in tumor tissues and peripheral blood derived from gastric adenocarcinoma patients," Cell Research, vol. 22, no. 1, pp. 248-258, 2012.

[19] A. Marusyk, D. P. Tabassum, P. M. Altrock, V. Almendro, F. Michor, and K. Polyak, "Non-cell-autonomous driving of tumour growth supports sub-clonal heterogeneity," Nature, vol. 514, no. 7520, pp. 54-58, 2014.

[20] T. Shibue and R. A. Weinberg, "EMT, CSCs, and drug resistance: the mechanistic link and clinical implications," Nature Reviews. Clinical Oncology, vol. 14, no. 10, pp. 611-629, 2017.

[21] D. Ribatti, "Epithelial-mesenchymal transition in morphogenesis, cancer progression and angiogenesis," Experimental Cell Research, vol. 353, no. 1, pp. 1-5, 2017.

[22] X. Z. Chen, X. H. Song, X. L. Chen, C. C. Li, X. M. Mo, and J. K. $\mathrm{Hu}$, "A bottleneck in understanding metastatic cancer stem cell of peritoneal seeding from gastric cancer: a null result in brief," Journal of Cancer, vol. 8, no. 16, pp. 3274-3277, 2017.

[23] C. Zhang, C. Li, F. He, Y. Cai, and H. Yang, "Identification of CD44+CD24+ gastric cancer stem cells," Journal of Cancer Research and Clinical Oncology, vol. 137, no. 11, pp. 16791686, 2011.

[24] X. Zhang, R. Hua, X. Wang et al., "Identification of stem-like cells and clinical significance of candidate stem cell markers in gastric cancer," Oncotarget, vol. 7, no. 9, pp. 9815-9831, 2016.

[25] Z. F. Miao, H. Xu, H. M. Xu et al., "DLL4 overexpression increases gastric cancer stem/progenitor cell self-renewal ability and correlates with poor clinical outcome via Notch-1 signaling pathway activation," Cancer Medicine, vol. 6, no. 1, pp. 245-257, 2017.

[26] X. L. Chen, X. Z. Chen, Y. G. Wang et al., "Clinical significance of putative markers of cancer stem cells in gastric cancer: a retrospective cohort study," Oncotarget, vol. 7, no. 38, pp. 6204962069, 2016.

[27] S. Fujita, H. Suzuki, M. Kinoshita, and S. Hirohashi, "Inhibition of cell attachment, invasion and metastasis of human carcinoma cells by anti-integrin beta 1 subunit antibody," Japanese Journal of Cancer Research, vol. 83, no. 12, pp. 1317-1326, 1992.

[28] Y. Ishii, A. Ochiai, T. Yamada et al., "Integrin $\alpha 6 \beta 4$ as a suppressor and a predictive marker for peritoneal dissemination in human gastric cancer," Gastroenterology, vol. 118, no. 3, pp. 497-506, 2000.

[29] H. Kotanagi, Y. Satto, N. Shiozawa, and K. Koyama, "Establishment of a human cancer cell line with high potential for peritoneal dissemination," Journal of Gastroenterology, vol. 30, no. 3, pp. 437-438, 1995.

[30] K. Kaneko, M. Yano, T. Tsujinaka et al., "Establishment of a visible peritoneal micrometastatic model from a gastric adenocarcinoma cell line by green fluorescent protein," International Journal of Oncology, vol. 16, no. 5, pp. 893-898, 2000. 
[31] H. Nishimori, T. Yasoshima, R. Denno et al., "A novel experimental mouse model of peritoneal dissemination of human gastric cancer cells: different mechanisms in peritoneal dissemination and hematogenous metastasis," Japanese Journal of Cancer Research, vol. 91, no. 7, pp. 715-722, 2000.

[32] L. Jia, S. Ren, T. Li et al., "Effects of combined simultaneous and sequential endostar and cisplatin treatment in a mice model of gastric cancer peritoneal metastases," Gastroenterology Research and Practice, vol. 2017, Article ID 2920384, 7 pages, 2017.

[33] K. Yanagihara, M. Takigahira, H. Tanaka et al., "Development and biological analysis of peritoneal metastasis mouse models for human scirrhous stomach cancer," Cancer Science, vol. 96, no. 6, pp. 323-332, 2005.

[34] P. Ray, H. Pimenta, R. Paulmurugan et al., "Noninvasive quantitative imaging of protein-protein interactions in living subjects," Proceedings of the National Academy of Sciences of the United States of America, vol. 99, no. 5, pp. 3105-3110, 2002.

[35] P. Ciana, M. Raviscioni, P. Mussi et al., "In vivo imaging of transcriptionally active estrogen receptors," Nature Medicine, vol. 9, no. 1, pp. 82-86, 2003.

[36] N. Yamamoto, P. Jiang, M. Yang et al., "Cellular dynamics visualized in live cells in vitro and in vivo by differential dual-color nuclear-cytoplasmic fluorescent-protein expression," Cancer Research, vol. 64, no. 12, pp. 4251-4256, 2004.

[37] R. M. Hoffman, "The multiple uses of fluorescent proteins to visualize cancer in vivo_," Nature Reviews. Cancer, vol. 5, no. 10, pp. 796-806, 2005.

[38] T. Furukawa, X. Fu, T. Kubota, M. Watanabe, M. Kitajima, and R. M. Hoffman, "Nude mouse metastatic models of human stomach cancer constructed using orthotopic implantation of histologically intact tissue," Cancer Research, vol. 53, no. 5, pp. 1204-1208, 1993.

[39] Y. Jia, M. Liu, W. Huang et al., "Recombinant human endostatin endostar inhibits tumor growth and metastasis in a mouse xenograft model of colon cancer," Pathology Oncology Research, vol. 18, no. 2, pp. 315-323, 2012.

[40] B. K. Abraham, P. Fritz, M. McClellan, P. Hauptvogel, M. Athelogou, and H. Brauch, "Prevalence of CD44+/CD24/low cells in breast cancer may not be associated with clinical outcome but may favor distant metastasis," Clinical Cancer Research, vol. 11, no. 3, pp. 1154-1159, 2005.

[41] F. Li, B. Tiede, J. Massagué, and Y. Kang, "Beyond tumorigenesis: cancer stem cells in metastasis," Cell Research, vol. 17, no. 1, pp. 3-14, 2007.

[42] C. Ginestier, M. H. Hur, E. Charafe-Jauffret et al., "ALDH1 is a marker of normal and malignant human mammary stem cells and a predictor of poor clinical outcome," Cell Stem Cell, vol. 1, no. 5, pp. 555-567, 2007.

[43] R. Liu, X. Wang, G. Y. Chen et al., "The prognostic role of a gene signature from tumorigenic breast-cancer cells," The New England Journal of Medicine, vol. 356, no. 3, pp. 217226, 2007.

[44] C. Sheridan, H. Kishimoto, R. K. Fuchs et al., "CD44+/CD24breast cancer cells exhibit enhanced invasive properties: an early step necessary for metastasis," Breast Cancer Research, vol. 8 , no. $5,2006$.

[45] P. C. Hermann, S. L. Huber, T. Herrler et al., "Distinct populations of cancer stem cells determine tumor growth and metastatic activity in human pancreatic cancer," Cell Stem Cell, vol. 1, no. 3, pp. 313-323, 2007.
[46] R. Pang, W. L. Law, A. C. Y. Chu et al., "A subpopulation of $\mathrm{CD}^{2} 6^{+}$cancer stem cells with metastatic capacity in human colorectal cancer," Cell Stem Cell, vol. 6, no. 6, pp. 603-615, 2010.

[47] M. Diehn and R. Majeti, "Metastatic cancer stem cells: an opportunity for improving cancer treatment?," Cell Stem Cell, vol. 6 , no. 6, pp. 502-503, 2010.

[48] K. Giehl, B. Skripczynski, A. Mansard, A. Menke, and P. Gierschik, "Growth factor-dependent activation of the Ras-Raf-MEK-MAPK pathway in the human pancreatic carcinoma cell line PANC-1 carrying activated K-ras: implications for cell proliferation and cell migration," Oncogene, vol. 19, no. 25, pp. 2930-2942, 2000.

[49] C. Simon, M. J. Hicks, A. J. Nemechek et al., "PD 098059, an inhibitor of ERK1 activation, attenuates the in vivo invasiveness of head and neck squamous cell carcinoma," British Journal of Cancer, vol. 80, no. 9, pp. 1412-1419, 1999.

[50] H. Acloque, M. S. Adams, K. Fishwick, M. Bronner-Fraser, and M. A. Nieto, "Epithelial-mesenchymal transitions: the importance of changing cell state in development and disease," The Journal of Clinical Investigation, vol. 119, no. 6, pp. 14381449, 2009.

[51] T. Brabletz, "EMT and MET in metastasis: where are the cancer stem cells?," Cancer Cell, vol. 22, no. 6, pp. 699-701, 2012.

[52] A. F. Vieira and J. Paredes, "P-cadherin and the journey to cancer metastasis," Molecular Cancer, vol. 14, no. 1, 2015.

[53] A. S. Ribeiro, B. Sousa, L. Carreto et al., "P-cadherin functional role is dependent on E-cadherin cellular context: a proof of concept using the breast cancer model," The Journal of Pathology, vol. 229, no. 5, pp. 705-718, 2013.

[54] J. P. Thiery and J. P. Sleeman, "Complex networks orchestrate epithelial-mesenchymal transitions," Nature Reviews. Molecular Cell Biology, vol. 7, no. 2, pp. 131-142, 2006.

[55] J. M. Lee, S. Dedhar, R. Kalluri, and E. W. Thompson, "The epithelial-mesenchymal transition: new insights in signaling, development, and disease," The Journal of Cell Biology, vol. 172, no. 7, pp. 973-981, 2006.

[56] M. Al-Hajj, M. S. Wicha, A. Benito-Hernandez, S. J. Morrison, and M. F. Clarke, "Prospective identification of tumorigenic breast cancer cells," Proceedings of the National Academy of Sciences of the United States of America, vol. 100, no. 7, pp. 3983-3988, 2003.

[57] L. Li and W. B. Neaves, "Normal stem cells and cancer stem cells: the niche matters," Cancer Research, vol. 66, no. 9, pp. 4553-4557, 2006.

[58] M. Mladinich, D. Ruan, and C. H. Chan, "Tackling cancer stem cells via inhibition of EMT transcription factors," Stem Cells International, vol. 2016, Article ID 5285892, 10 pages, 2016.

[59] C. L. Chaffer, J. P. Brennan, J. L. Slavin, T. Blick, E. W. Thompson, and E. D. Williams, "Mesenchymal-to-epithelial transition facilitates bladder cancer metastasis: role of fibroblast growth factor receptor-2," Cancer Research, vol. 66, no. 23, pp. 11271-11278, 2006.

[60] F. Hlubek, "Wnt/FZD signaling and colorectal cancer morphogenesis," Frontiers in Bioscience, vol. 12, no. 1, pp. 458470, 2007.

[61] C. C. Yates, C. R. Shepard, D. B. Stolz, and A. Wells, "Co-culturing human prostate carcinoma cells with hepatocytes leads to increased expression of E-cadherin," British Journal of Cancer, vol. 96, no. 8, pp. 1246-1252, 2007. 
[62] H. Hugo, M. L. Ackland, T. Blick et al., "Epithelial-mesenchymal and mesenchymal-epithelial transitions in carcinoma progression," Journal of Cellular Physiology, vol. 213, no. 2, pp. 374-383, 2007.

[63] C. L. Chaffer, B. Dopheide, D. R. McCulloch et al., "Upregulated MT1-MMP/TIMP-2 axis in the TSU-Pr1-B1/B2 model of metastatic progression in transitional cell carcinoma of the bladder," Clinical \& Experimental Metastasis, vol. 22, no. 2, pp. 115-125, 2005.

[64] J. H. Tsai, J. L. Donaher, D. A. Murphy, S. Chau, and J. Yang, "Spatiotemporal regulation of epithelial-mesenchymal transition is essential for squamous cell carcinoma metastasis," Cancer Cell, vol. 22, no. 6, pp. 725-736, 2012.

[65] O. H. Ocaña, R. Córcoles, A. Fabra et al., "Metastatic colonization requires the repression of the epithelial-mesenchymal transition inducer Prrxl," Cancer Cell, vol. 22, no. 6, pp. 709-724, 2012.

[66] J. P. Thiery, "Epithelial-mesenchymal transitions in tumour progression," Nature Reviews. Cancer, vol. 2, no. 6, pp. 442454, 2002.

[67] J. P. Thiery, H. Acloque, R. Y. J. Huang, and M. A. Nieto, "Epithelial-mesenchymal transitions in development and disease," Cell, vol. 139, no. 5, pp. 871-890, 2009.

[68] R. Kalluri and R. A. Weinberg, "The basics of epithelialmesenchymal transition," The Journal of Clinical Investigation, vol. 119, no. 6, pp. 1420-1428, 2009.

[69] M. K. Jolly, "Implications of the hybrid epithelial/mesenchymal phenotype in metastasis," Frontiers in Oncology, vol. 5, 2015.

[70] M. K. Jolly, S. C. Tripathi, D. Jia et al., "Stability of the hybrid epithelial/mesenchymal phenotype," ONCOTARGET, vol. 7, no. 19, pp. 27067-27084, 2016.

[71] M. K. Jolly, D. Jia, S. C. Tripathi et al., "Stability and stemness of the hybrid epithelial-mesenchymal phenotype," Cancer Research, vol. 7713, 2017.

[72] M. K. Jolly, S. A. Mani, and H. Levine, "Hybrid epithelial/mesenchymal phenotype(s): The 'fittest' for metastasis?" Biochimica et Biophysica Acta (BBA) - Reviews on Cancer, vol. 1870, no. 2, pp. 151-157, 2018.

[73] F. Bocci, L. Gearhart-Serna, M. Boareto et al., "Toward understanding cancer stem cell heterogeneity in the tumor microenvironment," Proceedings of the National Academy of Sciences of the United States of America, vol. 116, no. 1, pp. 148-157, 2019.

[74] T. Yin, H. Wei, S. Gou et al., "Cancer stem-like cells enriched in Panc-1 spheres possess increased migration ability and resistance to gemcitabine," International Journal of Molecular Sciences, vol. 12, no. 3, pp. 1595-1604, 2011.

[75] T. M. Yeung, S. C. Gandhi, J. L. Wilding, R. Muschel, and W. F. Bodme, "Cancer stem cells from colorectal cancer-derived cell lines," Proceedings of the National Academy of Sciences, vol. 107, no. 8, pp. 3722-3727, 2010.

[76] J. Sheridan, C. A. Coe, P. Doran et al., "Protocol for a multicentred randomised controlled trial investigating the use of personalised golimumab dosing tailored to inflammatory load in ulcerative colitis: the GOAL-ARC study (GLM dose Optimisation to Adequate Levels to Achieve Response in Colitis) led by the INITIAtive group (NCT 0268772)," BMJ Open Gastroenterology, vol. 5, no. 1, p. e000174, 2018.

[77] B.-T. Preca, K. Bajdak, K. Mock et al., "A novel ZEB1/HAS2 positive feedback loop promotes EMT in breast cancer," ONCOTARGET, vol. 8, no. 7, pp. 11530-11543, 2017.
[78] S. M. Mooney, V. Talebian, M. K. Jolly et al., "The GRHL2/ZEB feedback loop-a key axis in the regulation of EMT in breast cancer," Journal of Cellular Biochemistry, vol. 118, no. 9, pp. 2559-2570, 2017.

[79] B. De Craene and G. Berx, "Regulatory networks defining EMT during cancer initiation and progression," Nature Reviews Cancer, vol. 13, no. 2, pp. 97-110, 2013.

[80] H. Roca, J. Hernandez, S. Weidner et al., “Transcription factors OVOL1 and OVOL2 induce the mesenchymal to epithelial transition in human cancer," PLOS ONE, vol. 8, no. 10, article e76773, 2013.

[81] M. Lu, M. K. Jolly, H. Levine, J. N. Onuchic, and E. Ben-Jacob, "MicroRNA-based regulation of epithelial-hybrid-mesenchymal fate determination," Proceedings of the National Academy of Sciences, vol. 110, no. 45, pp. 18144-18149, 2013.

[82] Q. Xu, F. Deng, Y. Qin et al., "Long non-coding RNA regulation of epithelial-mesenchymal transition in cancer metastasis," Cell Death \& Disease, vol. 7, no. 6, article e2254, 2016.

[83] R. L. Brown, L. M. Reinke, M. S. Damerow et al., "CD44 splice isoform switching in human and mouse epithelium is essential for epithelial-mesenchymal transition and breast cancer progression," JOURNAL OF CLINICAL INVESTIGATION, vol. 121, no. 3, pp. 1064-1074, 2011.

[84] B.-T. Preca, K. Bajdak, K. Mock et al., "A self-enforcing CD44s/ZEB1 feedback loop maintains EMT and stemness properties in cancer cells," International Journal of Cancer, vol. 137, no. 11, pp. 2566-2577, 2015.

[85] M. K. Jolly, B.-T. Preca, S. C. Tripathi et al., "Interconnected feedback loops among ESRP1, HAS2, and CD44 regulate epithelial-mesenchymal plasticity in cancer," APL BIOENGINEERING, vol. 2, no. 3, p. 031908, 2018.

[86] W. L. Tam and R. A. Weinberg, "The epigenetics of epithelialmesenchymal plasticity in cancer," NATURE MEDICINE, vol. 19, no. 11, pp. 1438-1449, 2013.

[87] A. G. de Herreros, S. Peiró, M. Nassour, and P. Savagner, "Snail family regulation and epithelial mesenchymal transitions in breast cancer progression," Journal of Mammary Gland Biology and Neoplasia, vol. 15, no. 2, pp. 135-147, 2010. 